\title{
Spontaneous coronary artery dissection in the postpartum period: association with antiphospholipid antibody
}

\section{Krishnamurthy, R Desai, H Patel}

Heart 2004;90:e53 (http://www.heartinl.com/cgi/content/full/90/9/e53). doi: 10.1136/hrt.2004.038869

Spontaneous coronary artery dissection (SCAD) is an extremely uncommon cause of myocardial infarction, occurring predominantly in women during or after pregnancy. The exact aetiology is unknown. This report describes a 33 year postpartum woman with diagnosed SCAD who tested positive for anticardiolipin antibody. This is the first case of SCAD in a patient with antiphospholipid antibody. The authors hypothesised that there should be a strong association between them.

A 31 year African American woman, G5P2032 (gravida 5 , para 2, spontaneous abortion 0 , induced abortions 3, live births 2), was discharged home after an uneventful full term vaginal delivery of a healthy baby. Eight days post partum she was admitted with substernal chest tightness. She had no known risk factors for coronary artery disease and no history of any connective tissue disorder. She did not smoke or use alcohol, drugs, or oral contraceptive. There was no history of sudden death in her family. Her pregnancy course had been uncomplicated. Her three abortions were elective and they predated her two normal pregnancies.

On examination, she was an apparently healthy woman with heart rate of 74 beats/min and a blood pressure of 124/ $71 \mathrm{~mm} \mathrm{Hg}$. Her physical examination results were within normal limits.

ECG showed ST segment elevation in leads Vl-V3 and symmetric $\mathrm{T}$ inversion from Vl-V6. Cardiac enzymes were abnormal with a peak troponin I of $18.6 \mathrm{ng} / \mathrm{ml}$ and creatine kinase $\mathrm{MB}$ fraction index of 8.8 , both at 16 hours after admission. Echocardiogram showed mild hypokinesis of distal septal and apical segment of the left ventricle.

The patient was thoroughly investigated for risk factors for acute myocardial infarction (AMI), which were negative. Urine toxicology was also negative. However, she tested positive for anticardiolipin antibody (IgM) at a value of 25 micro pulse lidar units (reference range 0-9) but did not exhibit any features to suggest antiphospholipid antibody syndrome (APS)

After three days of treatment with heparin, glyceryl trinitrate, $\beta$ blocker, angiotensin converting enzyme inhibitor, and aspirin, the patient underwent cardiac catheterisation. It showed a dissection in the middle third of the left anterior descending coronary artery (fig 1). The other coronary arteries were normal. During the angiogram, the patient did not undergo any further intervention.

One year after the episode, the patient continues to be symptom-free and her activities are not limited.

\section{DISCUSSION}

AMI is uncommon under the age of 40 years, especially among women. This age group accounts for about $5.6 \%$ of all cases of AMI. Among them, women (under 40 years) account for just $0.7 \%$ of cases. ${ }^{1}$ There are identifiable risk factors in most instances.

Primary non-traumatic spontaneous coronary artery dissection (SCAD) is a rare cause of coronary artery disease in the younger age group. It was first described in a 42 year woman by Pretty in $1931 .^{2}$ In the pre-cardiac catheterisation era, the condition was usually diagnosed at necropsy. More than 150 cases have been reported in the literature so far. Of these about 60 cases have been confirmed by coronary angiography. ${ }^{3}$ On the basis of epidemiological data, the condition occurs in one in 20000 to 30000 deliveries and has a high mortality rate for both mother and child.

SCAD occurs most commonly in the third trimester of pregnancy and in the early postpartum period. The dissections involve the left main and left anterior descending arteries in about $80 \%$ of cases. The overall mortality is more than $50 \%$ at presentation. This improves to $85 \%$ survival for patients who survive the acute phase. ${ }^{4}$ In contrast to other causes of myocardial infarction, coronary artery dissection occurs in normal coronary arteries. It is also not associated with the classic coronary disease risk factors. There is no agreement on the optimal treatment of this condition. It is worth stressing that thrombolytic treatment is relatively

Abbreviations: AMI, acute myocardial infarction; APS, antiphospholipid antibody syndrome; SCAD, spontaneous coronary artery dissection

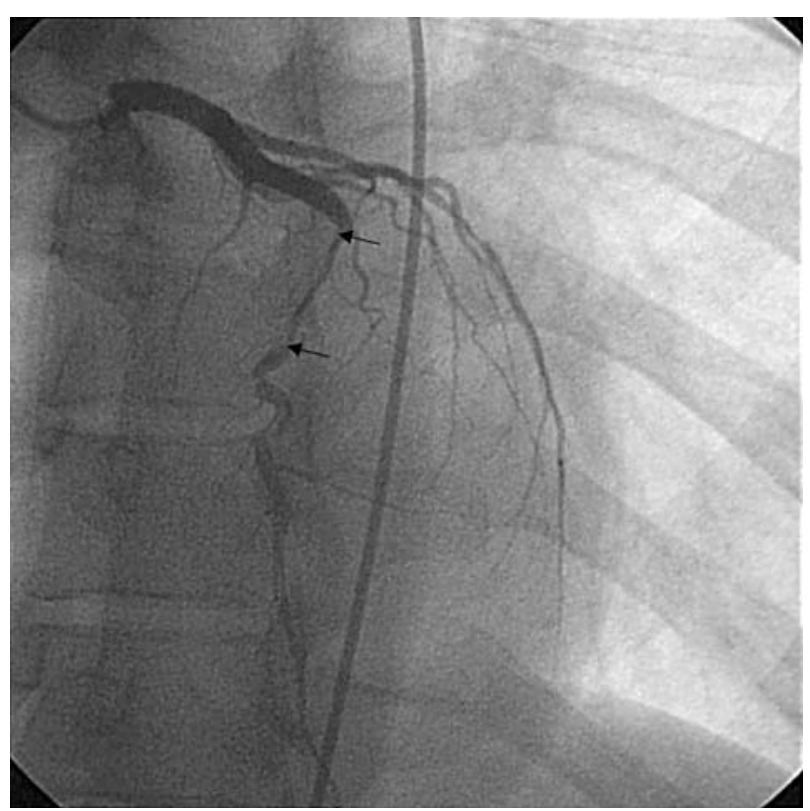

Figure 1 Left anterior oblique coronary angiogram showing the dissected segment of the left anterior descending coronary artery between the two black arrows. 


\section{LEARNING POINTS}

Pregnancy and postpartum related coronary dissections are important causes of acute myocardial infarction in the young. A high index of suspicion is needed, as thrombolytic treatment is relatively contraindicated in this setting.

Antiphosholipid antibodies may have a role in the pathogenesis of dissection by causing endothelial dysfunction, although such an association with spontaneous coronary artery dissection has not been reported.

contraindicated in this condition, as it may cause propagation of the dissection and expansion of the intramural haematoma. ${ }^{5}$

The aetiology of SCAD is unknown. The high incidence in pregnancy and puerperium indicate hormonal changes and haemodynamic stress as possible factors. Heefner ${ }^{6}$ hypothesised a two step process for the pathogenesis of SCAD. Firstly, the haemodynamic stress of pregnancy leads to an initial intimal rupture. This is followed by a delayed bleeding in the tunica media caused by the clotting changes that occur during the puerperium. ${ }^{6}$ A primary rupture of the vasa vasorum into the vessel wall has been noted in some cases. Cystic medial necrosis and other defects in collagen metabolism have also been described in some patients.

Interest in autoimmune causes of SCAD has centred on some reported cases of vasculitis and periadventitial infiltrates composed of histiocytes and eosinophilic granulocytes. Release of lytic substances by these cells was thought to damage collagen and elastin fibres, leading to coronary artery dissection.

The observed prevalence of AMI in APS is low, ranging from $4-7 \%$, and usually results from the association with systemic lupus erythematosus and premature atherosclerosis. ${ }^{7}$ There is no reported association between SCAD and APS. Nevertheless, coronary bypass graft and angioplasty occlusions have been associated with the presence of anticardiolipin antibody.

APS is characterised by recurrent arterial or venous thrombosis or fetal loss in the presence of antiphospholipid antibodies. The exact pathogenesis is unclear. Among the proposed mechanisms is the interaction between antiphospholipid antibodies and the surface membranes of cells involved in the coagulation cascade-namely, platelets and endothelial cells. ${ }^{8}$ Antiphospholipid antibodies cause endothelial perturbation and this may be related to altered regulation of the balance between mediators active on vessel dilatation or constriction (especially in the arterial tree). ${ }^{8}$

A correlation between antiphospholipid antibodies and SCAD has not been noted in the literature. However, antiphospholipid antibodies have been reported in two cases of carotid artery dissection. ${ }^{9}{ }^{10}$ In the light of these reports, further investigations are required to clarify the role of antiphospholipid antibodies in the causation of dissection.

\section{Authors' affiliations}

M Krishnamurthy, R Desai, H Patel, Department of Medicine, Wyckoff Heights Medical Center, Brooklyn, New York, USA

Affiliated to Weill Medical College of Cornell University, Ithaca, New York, USA

Correspondence to: Dr Mahesh Krishnamurthy, Wyckoff Heights Medical Center, Brooklyn, New York, USA; mahesh_kmurthy@ yahoo.com

Accepted 28 April 2004

\section{REFERENCES}

1 Badui E, Enciso R. Acute myocardial infarction during pregnancy and puerperium: a review. Angiology 1996;47:739-56.

2 Pretty HC. Dissecting aneurysm of a coronary artery in a woman aged 42. BMJ $1931 ; \mathrm{i}: 667$.

3 Koul AK, Hollander G, Moskovits N, et al. Coronary artery dissection during pregnancy and the postpartum period: two case reports and review of literature. Catheter Cardiovasc Interv 2001;52:88-94.

4 Engelman DT, Thayer J, Derossi J, et al. Pregnancy related coronary artery dissection: a case report and collective review. Conn Med 1993;57:135-9.

5 Klutstein MW, Tzivoni D, Bitran D, et al. Treatment of spontaneous coronary artery dissection: report of three cases. Cathet Cardiovasc Diagn 1997;40:372-6.

6 Heefner WA. Dissecting hematoma of the coronary artery: a possible complication of oral contraceptive therapy. JAMA 1973;223:550-1.

7 Adler Y, Kinkelstein Y, Zanderman-Goddard G, et al. The presence of antiphospholipid antibodies in acute myocardial infarction. Lupus 1995;4:309-13.

8 Meroni PL, Raschi E, Testoni C, et al. Antiphospholipid antibodies and the endothelium. Rheum Dis Clin North Am 2001;27:587-602.

9 Caso V, Paciaroni M, Parnetti L, et al. Stroke related to carotid artery dissection in a young patient with Takayasu arteritis, systemic lupus erythematosus and antiphospholipid antibody syndrome. Cerebrovasc Dis 2002; 13:67-9

10 Taillan B, Garnier G, Dujardin P. [Dissection of the internal carotid artery in antiphospholipid syndrome]. Rev Med Interne 1999;20:1048. 